\title{
Forced current sheet structure, formation and evolution: application to magnetic reconnection in the magnetosphere
}

\author{
V. I. Domrin and A. P. Kropotkin \\ Skobeltsyn Institute of Nuclear Physics, Moscow State University, 119992 Moscow, Russia \\ Received: 29 September 2003 - Revised: 1 May 2004 - Accepted: 28 May 2004 - Published: 14 July 2004 \\ Part of Special Issue "Spatio-temporal analysis and multipoint measurements in space"
}

\begin{abstract}
By means of a simulation model, the earlier predicted nonlinear kinetic structure, a Forced Kinetic Current Sheet (FKCS), with extremely anisotropic ion distributions, is shown to appear as a result of a fast nonlinear process of transition from a previously existing equilibrium. This occurs under triggering action of a weak MHD disturbance that is applied at the boundary of the simulation box. In the FKCS, current is carried by initially cold ions which are brought into the CS by convection from both sides, and accelerated inside the CS. The process then appears to be spontaneously self-sustained, as a MHD disturbance of a rarefaction wave type propagates over the background plasma outside the CS. Comparable to the Alfvénic discontinuity in MHD, transformation of electromagnetic energy into the energy of plasma flows occurs at the FKCS. But unlike the MHD case, "free" energy is produced here: dissipation should occur later, through particle interaction with turbulent waves generated by unstable ion distribution being formed by the FKCS action. In this way, an effect of magnetic field "annihilation" appears, required for fast magnetic reconnection. Application of the theory to observations at the magnetopause and in the magnetotail is considered.
\end{abstract}

Key words. Magnetosphere physics (magnetotail; plasma sheet; storms and substorms)

\section{Introduction}

In recent studies, much evidence has appeared as to the existence and evolution of thin current sheets (CS) in space plasmas. An impressive evidence of this kind relating to the Earth's magnetosphere was presented at the STAMMS conference (Orléans, France, May 2003).

Theoretically, it is generally accepted (e.g. Syrovatskii, 1971; Parker, 1979) that slow, quasi-static evolution of mag-

Correspondence to: A. P. Kropotkin

(apkrop@dec1.sinp.msu.ru) netized space plasma objects often leads to the formation of CS of relatively small thickness. Later development of the configuration is believed to involve transformation of stored magnetic energy into energy of plasma flows, and dissipation. Actually that means the formation of specific "dissipative structures" (Nicolis and Prigogine, 1977; Haken, 1983) and is a manifestation of self-organization in open nonlinear systems. The relevant particular nonlinear dynamics is still an open problem.

Small transverse scale of CS arising during quasi-static evolution may often mean that only its kinetic description is appropriate. On the other hand, we then can treat the CS locally as a one-dimensional structure.

In the best documented case of the Earth's magnetosphere, the processes of CS formation and thinning are substantial constituents of its global dynamics. It involves a phase of quasi-static evolution that is followed by a catastrophe of equilibrium (Kropotkin and Sitnov, 1997; Kropotkin, 2000; Kropotkin et al., 2002a, b). In the global equilibrium state, i.e. on the quasi-static evolution stage, stability of the system is presumably dependent on the current density in the nearEarth portion of the magnetotail CS, where a transition takes place from the dipole-like to the tail-like field line structure (transition region, TR). A typical feature of TR is an intense CS thinning during substorm disturbances (Sergeev et al., 1993; Baker et al., 1999). Before the substorm activation (onset) the process may be treated as slow, quasistatic (Kropotkin and Lui, 1995; Schindler 1998), but during the activation it should become much faster. We propose that it is then induced by a fast, explosion-like nonlinear process of current filamentation in the tearing mode that occurs in a somewhat more distant portion of the magnetotail (Kropotkin, 2000; Kropotkin et al., 2002a, b).

We argue that outside the nonlinear tearing instability site, the induced process of CS thinning eventually results in the formation of a specific kinetic structure, namely an anisotropic forced kinetic CS (FKCS), and inside that structure, transformation takes place of the magnetic energy 
stored in the magnetotail into the energy of ions accelerated inside such a CS. That structure provides merging of magnetic field lines, and thus facilitates the global effect of magnetic reconnection.

In recent papers by these authors and their co-workers (Kropotkin and Domrin, 1995, 1996, 1997; Kropotkin et al., 1997; Sitnov et al., 2000; Sitnov and Sharma, 2000) a theory of a stationary FKCS was constructed. Substantial earlier work started by the studies of Speiser, and continued in papers by Eastwood, Hill, Frankfort and Pellat, Burkhart et al., Holland and Chen, Pritchett and Coroniti, provided certain guidelines for those recent studies.

The theory of the FKCS as a regular nonlinear plasma structure is based on the existence of a specific quasiadiabatic invariant, corresponding to ion oscillations about the central plane, during their motion on Speiser orbits, and also on the relation of that invariant to the magnetic moment being the adiabatic invariant of the ion motion outside CS. The CS is presented as a self-consistent structure. A profile of the equilibrium sheet was determined, along with its dependence on features of the ion distribution function.

A solution exists if outside the CS the distribution function is highly anisotropic: there is a pair of counter-streaming ion flows along $\boldsymbol{B}$ with a relative velocity equal to $2 V_{A}$, $V_{A}=B_{0} / \sqrt{4 \pi m n_{c}}$ is the Alfvén speed. The characteristic scale of the structure is determined by the Larmor radius $\rho_{c}=\sqrt{2 T_{c} / m} / \Omega_{0}$, and the inertial length $\lambda_{c}$ of ions outside CS:

$\delta=\sqrt{\pi \rho} \frac{c}{e} \frac{T_{c}^{3 / 2} n_{c}^{1 / 6}}{B_{0}^{4 / 3}}=\rho_{c}^{4 / 3} \lambda_{c}^{-1 / 3}$

(ion flows outside the CS have shifted Maxwellian distributions with temperature $T_{c}, \Omega_{0}=e B_{0} / m c$ ).

In the real configuration, ions convecting in the $z$ direction towards the CS in the crossed $E_{y}$ and $B_{x}$ fields, have a nearzero $x$ component of the bulk velocity. At the same time, those ions that run away from the CS, having been accelerated by the electric field inside the CS, have the bulk velocity equal to $2 V_{A}$ in that direction. The corresponding outward energy flux may be evaluated, and it coincides with the inward Pointing flux value. That energy transformation is just a manifestation of magnetic merging in the CS.

A substantial question remained as to whether the FKCS can really arise as a result of thin CS evolution. In Sect. 2 we analyse the process of FKCS formation (Domrin and Kropotkin, 2002, 2003). To this end we have worked out a special particle code. In Sect. 3 we present arguments to substantiate the adopted model of fast initial disturbance, for the particular case of substorm activation, and for a later stage of time-dependent evolution, we present the results of a theory of spontaneous magnetic field merging self-sustained by the FKCS action. Section 4 is devoted to the general situation of a thin CS as a planar discontinuity in collisionless plasma, with counterstreaming flows allowed. FKCS is a particular case of such a situation. More general cases are discussed, along with analysis of corresponding observational evidence.
In the concluding Sect. 5, the results are discussed in a more general context of the fast magnetic reconnection problem.

\section{Numerical simulation: the problem formulation, the solution method, and the results}

We have worked out a one-dimensional model for numerical simulation of the CS evolution, in order to reveal the way in which a stationary FKCS type structure arises (Domrin and Kropotkin, 2002, 2003). Dynamics of the hot plasma ions belonging to the initial plasma sheet and of the surrounding cold plasma are described by kinetic equations. A solution for ions is found by means of a particle code; electrons form a massless cold background. Self-consistent electromagnetic fields are determined by a solution of the Maxwell equations.

In the initial stationary state the system consists of a hot plasma with the ion temperature $T$, forming a onedimensional Harris-type CS, a uniform, relatively cold plasma background (with parameters $n_{c}, T_{c} \ll T$ ), and the magnetic field $\boldsymbol{B}\left(B_{t}(z), 0, B_{n}\right)$. We set $B_{n} \ll B_{0}$, with $B_{0}$ being the tangential field $B_{t}$ at $z \gg L_{0}$, where $L_{0}$ is the characteristic scale of the Harris sheet. The plasma in CS is in equilibrium over the $z$ direction owing to the tangential field component $B_{t}$. Thickness of the simulation box (SB) $Z_{0}$ is much greater than $L_{0}$.

Evolution of the system is started by a MHD disturbance; it is a fast magnetosonic wave incident on the CS in the normal direction. Far from the CS the incident wave is set weak, and it propagates with the Alfvén velocity $V_{A}$. The wave magnetic field is directed along the $\mathrm{x}$-axis while the electric field is in the $y$ direction.

At the SB boundary $Z_{0}$, the electric field of the incident wave, $E_{i}\left(Z_{0}, t\right)$ is set given: $E_{i}=0$ at $t<0, E_{i}=$ const $\neq 0$ at $t>0$. As a result of the wave interaction with the CS, a reflected wave appears, propagating outwards. At the $Z_{0}$ boundary, the electromagnetic field is a sum of fields of the incident and the reflected waves. Setting the boundary condition we assume that the reflected wave is weak as well; this identifies its velocity outside the SB, namely the Alfvén speed also, and the amplitude ratio of the wave electric and magnetic fields. The magnetic field $B_{t}$ at the second boundary of the $\mathrm{SB}, z=0$, is set equal to zero, due to symmetry. For the particle motion, conventional boundary conditions were adopted. The boundary condition involves both the inward convective ion flux and the outward freely escaping flux.

The translational symmetry over the $x, y$ directions is effectively used. Then the field equations may be reduced to an ordinary differential equation for the $y$ component of the vector potential. A full system of equations consists of equations of motion for separate particles, of a "material" equation coupling the current density and the electric field values, and of the Maxwell equations. Solutions of the equations are sought at particular instances (over a sequence of time steps with duration $\Delta t$ ). At every time step, $k \Delta t$, the number of particles is identified that are convected into the SB, and their distribution is generated. To calculate the smoothed 
current density $j_{1}$ at the spatial grid points, a linear weighting scheme is used. Then $j_{1}$ is inserted into a finite-difference equation for the vector potential. The equation is supplemented with conditions at both SB boundaries. Solution of the resulting set of algebraic equations is found by means of the Thomas algorithm. To solve the equations of motion of a particle, values of the magnetic and electric fields are recalculated at the particle position, by interpolation from the grid points. Then solving the equations of motion, the particle position in the phase space is determined at the next time moment $(k+1) \Delta t$.

The initial Harris sheet with the plasma (protons and electrons) density profile $n(z) / n(0)=\cosh ^{-2}\left(z / L_{0}\right)$ is in equilibrium with the magnetic field $B_{t}(z) / B_{0}=\tanh \left(z / L_{0}\right)$; here $n(0)=B_{0}^{2} / 8 \pi T$. The nonzero magnetic field component normal to the sheet is set $B_{n}=0.2 B_{0}$. The CS thickness is $L_{0}=\rho / 0.0587, \rho=(2 T / m)^{1 / 2} / \Omega_{0}$. The sheet is embedded in a uniform plasma with density which we have set equal to the maximum density of hot ions, $n_{c}=n(0)=N_{0}$; the background plasma temperature is set equal to $T_{C}=0.1 T$. The time step is taken equal to $0.1 \Omega_{0}^{-1}$. The SB size was $3 L_{0}$. The initial state of the system was modeled with 15000 macroparticles in the Harris sheet and 45000 macroparticles of cold background plasma. The code allows one to take into account the escape of accelerated particles from the $\mathrm{SB}$, and the influx of new ones across the $Z_{0}$ boundary, because of convection. Thus, the total particle number in the SB is not fixed in the course of evolution.

In Fig. 1 the dependence on $z$ is shown of the total (dimensionless) density $N(z) / N_{0}$, of the magnetic field intensity $B_{t}(z) / B_{0}$, and the electric field intensity $E(z) / E_{0}$ $\left(E_{0}=V_{A} B_{n} / c\right)$, for the time moments $t=1 / 10 \Omega_{0}, 5 / \Omega_{0}$, $10 / \Omega_{0}, 15 / \Omega_{0}, 20 / \Omega_{0}$, correspondingly. It is seen how in the uniform plasma a fast magnetosonic wave is propagating from the boundary $z=Z_{0}$, in the form of a weak collisionless shock. According to theory, peak-to-peak distance equal to the wavelength of the shock oscillatory front should be $2 \pi / \lambda_{c}$, where $\lambda_{c}$ is the ion inertial length. In simulation, this is valid with good accuracy. In theory also, in the background plasma the wave propagates with the Alfvén velocity $V_{A}$. An estimate based on simulation is in a very good consistency with theory as well. The plasma density and magnetic field near another boundary, $z=0$, stay undisturbed, and the electric field is nearly zero.

During the time $30 / \Omega_{0}<t<60 / \Omega_{0}$ the wave propagates from the boundary of the Harris sheet to its central plane. Later on, a reflected wave may be seen. In the $z=0$ vicinity, the electric field is then already nonzero. A sheet with enhanced plasma density and a sharp change in the magnetic field starts to form at $t \sim 80 / \Omega_{0}$.

At even later times, $t \sim 200 / \Omega_{0}$, in the vicinity of $z=0$ a thin embedded sheet has been formed. A structure is also seen which resembles the field profile in the initial Harris sheet. It stays there because of the hot plasma diamagnetism.

It is seen in Fig. 2, for $t>600 / \Omega_{0}$, that an almost uniform nonzero electric field has been established, and its magnitude changes only slightly. While it is seen that there is still

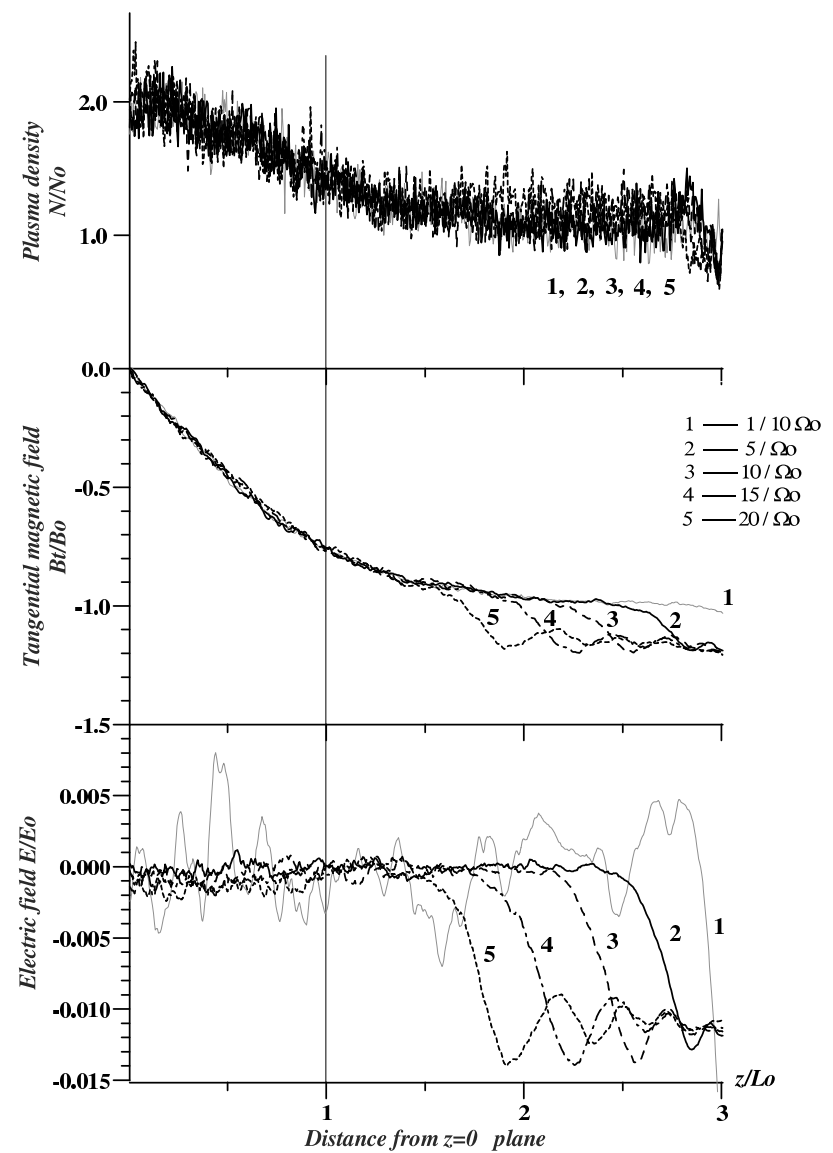

Fig. 1. Profiles of plasma density, the magnetic field tangential component, and the electric field, for initial moments of the CS evolution.

much hot plasma in the magnetic trap, it is now involved into the electric drift in the $x$ direction. At the same time, at $t \sim 650 / \Omega_{0}$, a balance is established of the ion convective flux into the SB, and the flux of accelerated ions outgoing along the field lines.

The established thin central CS is the same FKCS which is predicted by the theory, see Sect. 3. This is clear from comparison of the $B(z)$ profile with a theoretical profile calculated for the same value of the $v_{T c} / V_{A 0}=0.33$ parameter, taken from Sitnov et al. (2000), see Fig. 3. The sheet thickness estimates taken at the $B_{0} / 2$ level differ for large $t \sim 1000 / \Omega_{0}$ by several per cent only. In addition, according to the FKCS theory, we have $E / B_{n}=V_{A} / c$. In simulation, for $E$ we obtain $E / B_{n}=0.97 V_{A} / c$. Energy spectra have been calculated and compared with the theoretical spectrum. There is a good similarity in the spectrum profile for low energies (particles convecting slowly towards CS), and for high energies $\sim 40 \mathrm{keV}$ corresponding to ions accelerated inside the FKCS. 


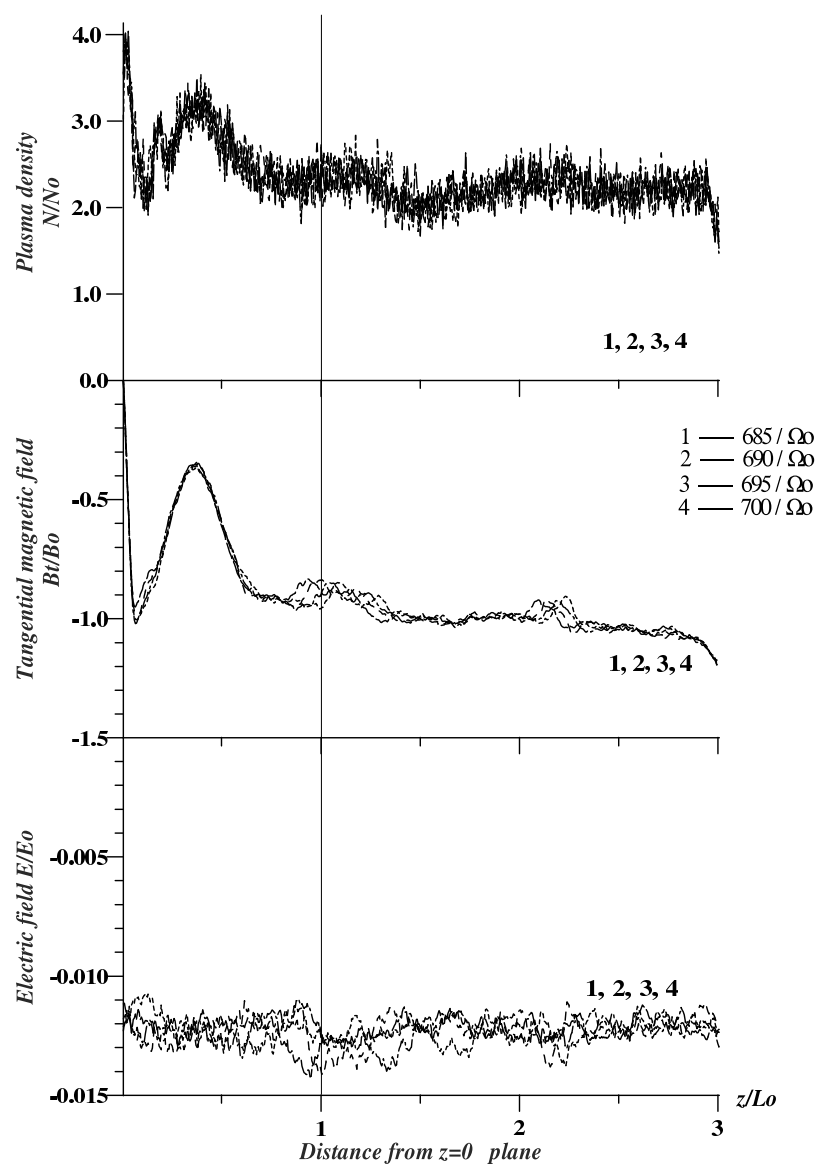

Fig. 2. The same profiles for $t>600 / \Omega_{0}$.

\section{Initial MHD evolution of a disturbance and sponta- neous magnetic field "annihilation" at a later stage}

How does a triggering MHD disturbance appear in the case of the geomagnetic tail, and what are the consequences? A tearing-type disturbance is proposed to occur somewhere in the near-Earth portion of the geomagnetic tail. At $B_{n} \neq 0$ and with nonzero background plasma density $n_{c}$ outside CS, the tearing mode occurs to be coupled with MHD modes. The disturbance propagates along the field lines towards the Earth. Propagating in a plasma with CS, such a disturbance gets deformed to a great extent. Near the CS, at small distances as compared to its scale along the Sun-Earth line, $L_{x}$, the disturbance is reduced to a pair of fast magnetosonic waves incident from both sides on the initial CS. Even if they are weak outside CS, as they interact with the CS, in the region with strongly depressed field $B$ inside the CS, the waves become nonlinear. Outside the CS reflected waves appear. The FKCS is being formed eventually.

In the initial disturbance, behind the weak shock fronts, plasma moves only in the perpendicular to CS direction (convection). At the nonlinear stage, ions are accelerated in the E-field inside the CS, are rotated in the $B_{n}$ field, and ejected from the CS along the magnetic field lines. In such a way, during the CS evolution, the plasma gets involved into mo-

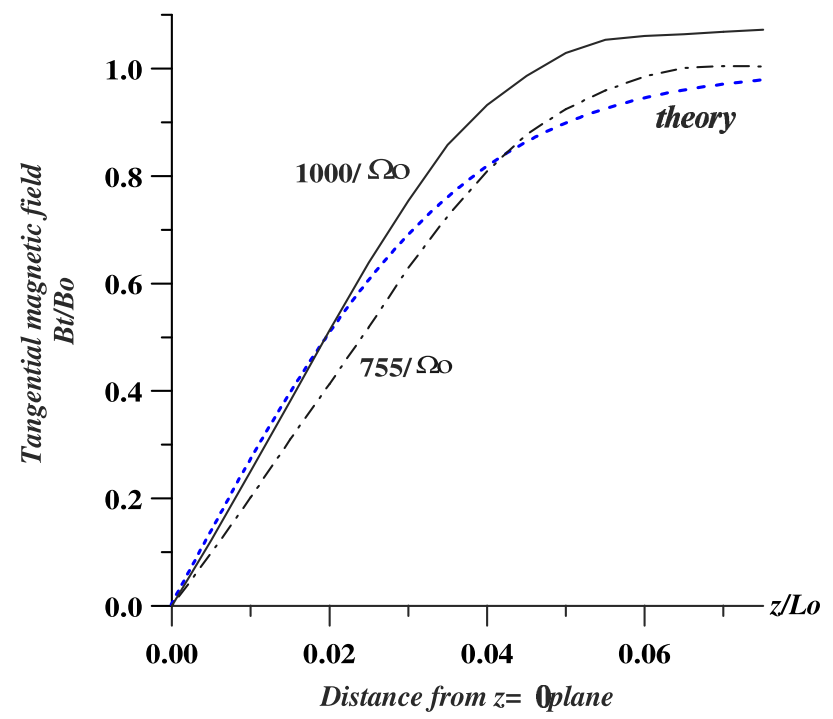

Fig. 3. Comparison of the magnetic field profile with the theoretical profile of the FKCS.

tion along the x-axis. And this is just the way a well-known nightside particle injection may appear during a substorm.

The above scenario of the global time-dependent structure involving the FKCS, may be analyzed in some more detail for later stages. Based on numerical simulation, a thin CS located in a background cold plasma, is known to be able in a short finite time to attain the specific features of the FKCS. A solution has been constructed (Domrin and Kropotkin, 2004) that joins several time-dependent zones with different plasma properties, see a schematic in Fig. 4. The extension to MHD of the gas dynamics rarefaction wave (zone III) joins the zone of undisturbed cold isotropic plasma at rest, existing at a large distance from the CS (zone IV) with the zone of convective isotropic plasma flow (zone II), where the convection velocity is determined by the above FKCS solution. The latter zone is linked with the expanding zone of ion anisotropy (zone I), where a pair of counterstreaming ion flows exists so that the net flux of matter is equal to zero. The double-stream zone, as it expands, is, however, always left behind the rear edge of the rarefaction wave running from the FKCS; so the presence of this zone does not affect the linkage of the two other zones, II and III.

In the rarefaction wave, the field $B_{t}$ decreases from $B_{0}$ down to $B_{1} \simeq B_{0}-B_{n}$. It may be thus argued that a time dependent solution has been found which describes spontaneous "annihilation" of the magnetic field in a plasma with a current sheet; it occurs following the transformation of the CS into FKCS.

\section{Planar discontinuities in collisionless plasma}

Observations carried out aboard a number of spacecraft while intersecting the magnetopause have revealed a complex structure of the CS forming the magnetopause, manifesting 
itself in the magnetic field variation during intersection. A complex and varying structure of the ion distribution function has also been found out; its most significant feature is a strong difference of that function from the thermodynamically equilibrium one. Outside the CS this is expressed in a multi-flow feature of the motion (Fuselier et al., 1991).

Such a difference is in general quite natural to expect for a relatively thin CS structure, in conditions typical for manifestation of collisionless kinetic effects. But in such conditions, for current-carrying discontinuities in a magnetized plasma, with a nonzero magnetic field normal component, classification existing in MHD (Landau and Lifshitz, 1974) should be modified. Along with the MHD rotational discontinuity, a structure of the FKCS is also now possible. It produces a transformation of the electromagnetic energy into the energy of a double-stream motion. In this way, a source of "free" energy appears for plasma turbulence development, with later dissipation, owing to the relaxation of the ion distribution on the turbulent waves. So a structure which much more resembles a rotational discontinuity than a shock, however, appears to be capable of dissipation of the electromagnetic field energy. By means of that structure, magnetic field "annihilation" can take place in collisionless space plasma.

Besides the limiting symmetric case corresponding to the FKCS, where the net bulk plasma flux across the discontinuity is absent, "intermediate" situations with multi-flow motion are also possible, with nonzero bulk flux but with transformation of electromagnetic energy into energy of counterstreaming ion flows still taking place.

Kinetic discontinuities with current, with the characteristic multi-flow ion motion on both sides of the CS, have been evidently observed at the dayside magnetopause. The observational data for a particular case (Avanov et al., 2001) indicate that there is a nonzero bulk ion flux, but smaller than for the MHD rotational discontinuity, across the discontinuity, while outside the CS the ion distribution function has a characteristic counterstreaming feature; this corresponds to the mentioned "intermediate" situation.

\section{Discussion and conclusion}

The possibilities of kinetic simulations are in general restricted, especially in the range of time and spatial scales: only a relatively small part of the magnetospheric system may be simulated, and on a relatively short time interval. In this situation, the choice of boundary conditions is crucial. In our approach, we point out the critical role played by a thin CS. On the one hand, a large amount of work, both in theory and in simulation, has been done (see, e.g. Birn et al., 2003 and references therein), which indicates that in processes of reconfiguration occurring in systems that globally are well described in ideal MHD, a thin CS should inevitably arise. At that point, MHD becomes no longer valid: a singularity appears in the current density distribution. On the other hand, those singularities are one-dimensional, in the sense that they appear as current sheets, with the spatial

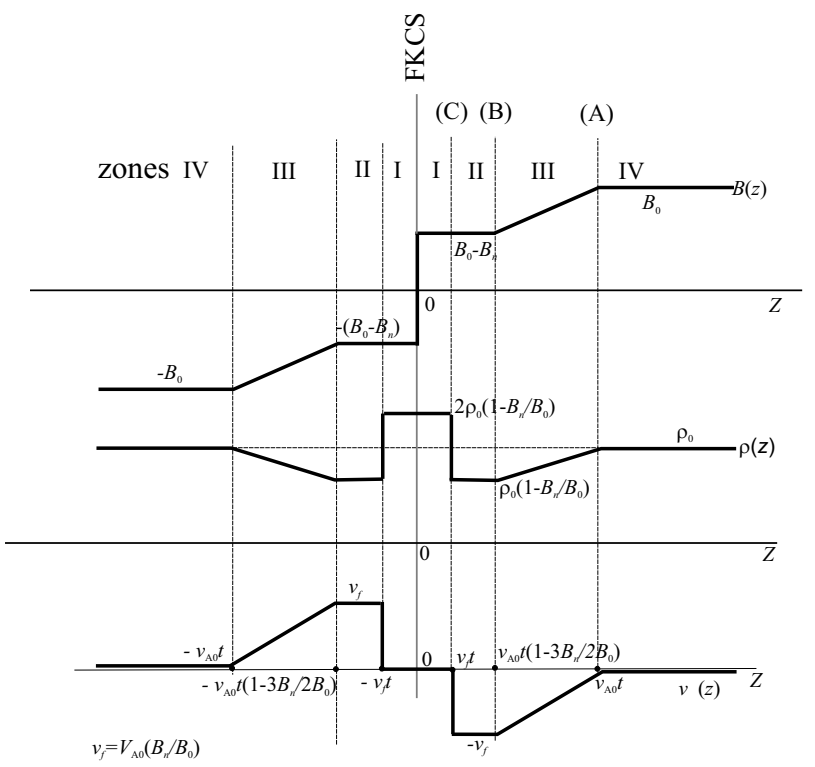

Fig. 4. Variations of density, magnetic field, and velocity in the plasma flows on both sides of the FKCS

scale over one direction, across the CS, being much smaller than over the other two. This greatly simplifies the problem of the KINETIC approach becoming necessary at that stage: spatial and temporal limitations arise quite naturally. Only kinetic processes occurring in such a "prepared" CS system are studied. They are considered to be started by a fast MHD disturbance appearing in the CS vicinity, in this case taking the form of one-dimensional plane waves. This means a specific formulation of boundary and initial conditions, and thus differs our study from the earlier work (see, e.g. Pritchett and Coroniti, 2002, and references therein). The appearance of the FKCS in our simulation means that specific kinetic effects come into play, involving nonadiabatic ion motions, strong anisotropy appearance, etc. This is totally different from the existing general situation: "The present simulations essentially exhibit fluid behavior. While it is reassuring that a collisionless kinetic plasma can behave like a fluid in its cross-field dynamics, the parallel dynamics for both waves and ballistic particle transport are not treated realistically" (Pritchett and Coroniti, 2002).

There is another problem with kinetic simulation of a dynamical process; it concerns the electrons. There are two aspects of that problem. First is the formal aspect of dealing with electrons in a hybrid code. They are treated as a massless cold background compensating the ion charge. Second is the underlying physical reasoning. If we speak of a stationary equilibrium configuration, then the electron distribution may be taken in any form which only satisfies the requirement of self-consistency in the collisionless Vlasov formulation. However, if we turn to the problem of time evolution, then we can do nothing more today than to follow it in the presented hybrid code manner, implicitly assuming that electrons, rather cold initially, cannot gain too much heating 
during the process of FKCS formation. The 1-D hybrid code is of course unable to reproduce any fast, generally 2-D or 3$\mathrm{D}$ processes, with electric fields involved, which could form wave turbulence producing electron (and ion) heating. What is, however, needed in order that the electron current could be comparable to the ion one, and thus substantially change the solution? It is easily shown that the electron temperature should then increase to the temperature of plasma sheet hot ions which form a pressure balance with the lobe magnetic field pressure. We just assume that this does not occur on rather short time scales involved. True, this might be verified theoretically; and that is one of the goals of future studies. By the way, note that a reliable solution of this problem in simulation needs a realistic particle-in-cell description of both species, i.e. that with a real electron-to-ion mass ratio, which is still unattainable in such simulations.

With the structure of the FKCS being established in the discussed way, it may be viewed as a "sink" for the convecting plasma flows, directed from both sides towards the central plane $z=0$. Indeed, as we have shown in Sect. 4, the flows of accelerated ions escaping the sheet along the field lines, and to which those convective flows are converted, have no influence on the pattern of the latter ones, and on the corresponding electric field $E_{y}$.

On the other hand, while the FKCS provides a mechanism responsible for spontaneous "annihilation" of the magnetic field, the magnetic field energy disappearing in a time unit, being transformed into the energy of ion flows, is given by the value of the Pointing vector,

$P=c \frac{E B_{t}}{4 \pi}=\frac{B_{t}^{2} B_{n}}{(4 \pi)^{3 / 2}\left(N_{0} m\right)^{1 / 2}}$.

To take an example, let us estimate the rate of energy transformation associated with a substorm activation in the geomagnetic tail. Take for this estimate $B_{t}=30 \mathrm{nT}$, $B_{n}=1 \mathrm{nT}, N_{0}=1 \mathrm{~cm}^{-3}$ in the hydrogen plasma, and we obtain $P=0,02 \mathrm{erg} \mathrm{s}^{-1} \mathrm{~cm}^{-2}$. Take into account that the activation process is localized, and assume that the localization scales over the $x$ and $y$ at the CS plane in the tail are equal to $3 R_{E}$. Then the rate of energy transformation is $10^{17} \mathrm{erg} / \mathrm{s}$. This estimate is not in contradiction to the available observation-based estimates of energy dissipation during substorms.

In general, the energy transformation takes place simultaneously over the whole CS surface, and this fact allows the transformed energy flux to be arbitrarily large. In that relation, the situation is similar to that of the Petschek (1964) MHD model, where the transformation occurs over a pair of slow shocks. However, as is the case there, the necessary magnetic reconnection, in the strict sense, i.e. as reconnection of magnetic field lines, does not occur on the CS itself. Such a reconnection can occur in a two-dimensional model, and not in a one-dimensional one which we are limited to. In the Petschek MHD model and in its time-dependent extension (Pudovkin and Semenov, 1985), the two-dimensional feature is expressed in the presence of the magnetic field neutral line, and of a diffusion region in its vicinity. The field line reconnection occurs right there, and it is provided by the finite conductivity. In collisionless plasma, on small scales comparable to the FKCS thickness, such a concept is not adequate. Presumably, in that case, the required dissipation is provided by the Landau damping during the tearing instability.

An important difference in the overall pattern is a consequence. In the MHD case, in the region of outer flow, the whole pattern of slow dependence on $(x, z, t)$ for the velocity field and the magnetic field may be determined, as the (in general, varying in time) rate of reconnection on the neutral line is postulated. To the contrary, in the collisionless situation, the reconnection rate at the neutral line is itself regulated by the large-scale dynamics of the system, being matched to it (see Kuznetsova et al., 2001, and references therein). Namely the characteristics of that large-scale dynamics are revealed in this paper: the above rate is determined by the rate of energy transformation (2).

Note here another difference from the MHD model (Pudovkin, Semenov, 1985), where an essentially twodimensional pattern of the outer zone flow appears from the very beginning. In the collisionless situation, first a fast nonlinear process may take the place of the FKCS formation and of the appearance of magnetic field annihilation at the sites of initially occurring extreme thinning of the equilibrium CS. Only later will that essentially one-dimensional process govern the rate of magnetic reconnection in the vicinity of the neutral line, as indicated by the cited studies involving numerical simulation of reconnection (Kuznetsova et al., 2001).

Acknowledgements. This work was supported by the Russian Foundation for Basic Research (project no. 02-05-64184).

Topical Editor T. Pulkkinen thanks two referees for their help in evaluating this paper.

\section{References}

Avanov, L. A., Smirnov, V. N., Waite Jr., J. H., Fuselier, S. A., and Vaisberg, O. L.: High-latitude magnetic reconnection in subAlfvenic flow: Interball Tail observations on May 29, 1996, J. Geophys. Res., 106, 29 491-29 502, 2001.

Baker, D. N., Pulkkinen, T. I., Büchner, J., and Klimas, A. J.: Substorms: A global instability of the magnetosphere-ionosphere system, J. Geophys. Res., 104, 14 601, 1999.

Birn, J., Schindler, K., and Hesse, M.: Formation of thin current sheets in the magnetotail: Effects of propagating boundary deformations, J. Geophys. Res., 108(A9), 1337, doi:10.1029/2002JA009641, 2003.

Domrin, V. I. and Kropotkin, A. P.: A kinetic model of thin current sheet generation and its role in magnetic reconnection in space plasmas. In: Proc. 6-th Int. Conf. on Substorms, Seattle, USA, 25-29 March 2002, 239-244, 2002.

Domrin, V. I. and Kropotkin, A. P.: Appearance of the kinetic current sheet in the geomagnetic tail: particle simulation, Geomagn. and Aeronomy, 43 (in Russian), 298-305, 2003.

Domrin, V. I. and Kropotkin, A. P.: Spontaneous magnetic field "annihilation" in space plasmas (in Russian), Geomagn. and Aeronomy, 44, 182-188, 2004. 
Fuselier, S. A., Klumpar, D. M., and Shelley, E. G.: Ion reflection and transmission during reconnection at the Earth's subsolar magnetopause, Geophys. Res. Lett., 18, 139, 1991.

Haken, H.: Synergetics: An Introduction, Springer-Verlag, New York, 1983.

Kropotkin, A. P.: Explosion-type dynamics of the magnetotail current sheet: role of mode coupling in substorm onsets, in Proc. 5-th Int. Conf. on Substorms, St. Petersburg, Russia, 16-20 May 2000 (ESA SP-443, July 2000), 127, 2000.

Kropotkin, A. P. and Domrin, V. I.: Ion dynamics and induced reconnection in a thin plasma sheet, Geomagn. and Aeronomy, 35, 1, (in Russian), 1995.

Kropotkin, A. P. and Domrin, V. I.: Theory of a thin onedimensional current sheet in collisionless space plasma, J. Geophys. Res., 101, 19893, 1996.

Kropotkin, A. P. and Domrin, V. I.: Ion distribution function in a thin one-dimensional plasma sheet, Geomagn. and Aeronomy, (in Russian), 37, 11, 1997.

Kropotkin, A. P. and Lui, A. T. Y.: Quasi-static evolution of the magnetosphere: The substorm growth phase, J. Geophys. Res., 100, $17231,1995$.

Kropotkin, A. P. and Sitnov, M. I.: Magnetospheric substorm dynamics from the viewpoint of open nonlinear systems theory, in: Problems of Geospace, edited by Pudovkin, M. I., Besser, B. P., Riedler, W., and Lyatskaya, A. M., Verlag Osterreich. Akad. Wiss., Wien, p. 75, 1997.

Kropotkin, A. P., Malova, H. V., and Sitnov, M. I.: The selfconsistent structure of a thin anisotropic current sheet, J. Geophys. Res., 102, 22 099, 1997.

Kropotkin, A. P., Trubachev, O. O., and Schindler, K.: Nonlinear mechanisms of a substorm explosion in the magnetotail, Geomag. and Aeronomy (English translation), 42, 277, 2002a.

Kropotkin, A. P., Trubachev, O. O., and Schindler, K.,: Substorm onset: a fast restructuring of the magnetotail associated with explosion-like turbulence enhancement, Geomag. and Aeronomy, (English translation), 42, 286, 2002b.
Kuznetsova, M. M. and Hesse, M.: Collisionless reconnection supported by nongyrotropic pressure effects in hybrid and particle simulations, J. Geophys. Res., 106, 3799-3810, 2001.

Landau, L. D. and Lifshitz, E. M.: Course of Theoretical Physics, vol. 8, Electrodynamics, Addison-Wesley-Longman-Reading, Mass., 1974.

Nicolis, G. and Prigogine, I.: Self-organization in nonequilibrium systems, John Wiley and Sons, New York, 1977.

Parker, E. N.: Cosmical magnetic fields, Oxford University Press, 1979.

Petschek, H. E.: Magnetic field annihilation, NASA Spec. Publ., SP-50, 425, 1964.

Pritchett, P. L. and Coroniti, F. V.: The challenge for kinetic simulations of substorm growth and onset, In: Proc. 6-th Int. Conf. on Substorms, Seattle, USA, 25-29 March 2002, 189-196, 2002.

Pudovkin, M. I. and Semenov, V. S.: Magnetic field reconnection theory and solar wind-magnetosphere interaction: A review, Space Sci. Rev., 41, 1-89, 1985.

Schindler, K.: Formation of thin current sheets and other recent developments in magnetospheric activity, Astrophys. Space Sci., 264, 289, 1998.

Sergeev, V. A., Mitchell, D. G., Russell, C. T., and Williams, D. J.: Structure of the tail plasma/current sheet at $\sim 11 R_{E}$ and its changes in the course of a substorm, J. Geophys. Res., 98, 17345 , 1993.

Sitnov, M. I. and Sharma, A. S.: Magnetotail thin current sheet models and their role in substorm physics, in: Proc. 5-th Int Conf. on Substorms, St. Petersburg, Russia, 16-20 May 2000 (ESA SP-443, July 2000), 113, 2000.

Sitnov, M. I., Zelenyi, L. M., Malova, Ch. V., and Sharma, A. S.: Thin current sheet embedded within a thicker plasma sheet: selfconsistent kinetic theory, J. Geophys. Res., 105, 13 029, 2000.

Syrovatskii, S. I.: On generation of a current sheet in a plasma with frozen-in magnetic field, Soviet JETP, 60, (in Russian), 1727, 1971. 